\title{
Management of Acute Opioid Toxicity in the Outpatient Setting
}

\author{
H. Harris Reynolds, MD; Timothy J. Orlowski, MD; Conway C. Huang, MD; C. Blake Phillips, MD
}

\section{PRACTICE POINTS}

- Opioid overdose continues to be a major public health concern. Dermatologists may encounter opioid toxicity in their practice, and prompt recognition and treatment are crucial.

- Naloxone is a quick-acting, easy-to-use, and relatively inexpensive medication that can easily be stored and administered in dermatologists' offices. $\square$ ermatologists' offices are not immune from potentially fatal medical events. As a result, it is imperative that dermatologists are well versed in how to manage emergency situations in an outpatient setting. We discuss signs, symptoms, and management of opioid toxicity with an instructive case from our outpatient, hospital-based dermatology clinic.

A 55-year-old woman presented for Mohs micrographic surgery for a large recurrent basal cell carcinoma on the right medial cheek. After informed consent was obtained and the procedure was discussed with the patient, she took one $0.5-\mathrm{mg}$ tablet of clonazepam for perioperative anxiety, which was part of her standard home medication regimen and preoperative administration of clonazepam had been discussed with the treating physician prior to her appointment. During tissue processing, the patient waited alone in the procedure room, with nursing checks every 10 to 15 minutes. Roughly 30 minutes after the initial stage was taken and clear margins were confirmed, the patient was found to be somnolent and unresponsive to voice, light, or touch. Physical examination revealed pupillary constriction, labored breathing, and absent blink reflex. Subsequent examination of the arms, which initially were covered by sleeves, revealed track marks. She was only aroused by a deep sternal rub, which caused her to moan and open her eyes. Her vital signs remained stable, with oxygen saturation greater than $90 \%$ and respiratory rate greater than 12 breaths per minute, and a registered nurse remained at her bedside to monitor her clinical status and vitals. Because this event took place in a hospital setting and the patient adequately maintained her airway, respiratory rate, and oxygenation status, the decision was made to closely observe the patient in our clinic. Without additional intervention, the patient gradually regained full awareness, orientation, and mental capacity over the course of 90 minutes. She was ambulatory and conversant at the completion of the procedure, and she declined additional screening for drug abuse or transfer to an acute care facility. She elected for discharge and was accompanied by a family member to drive her home. Later, a search of the state's prescription monitoring service revealed she had multiple prescriptions from numerous providers for benzodiazepines

Drs. Reynolds, Huang, and Phillips are from the University of Alabama at Birmingham. Dr. Orlowski is from the 479th Flying Training Group, Aviation Medicine Department, Naval Hospital Pensacola, Florida.

The authors report no conflict of interest.

The views expressed are those of the authors and are not to be construed as official or as representing those of the US Air Force or the Department of Defense.

Correspondence: H. Harris Reynolds, MD, John N. Whitaker Building, Ste 3400, 500 22nd St S, Birmingham, AL 35233-3110

(hharrisreynolds@gmail.com).

doi: 10.12788/cutis.0037 
and opioids. We suspect that her intoxication was the result of ingestion or injection of an opioid medication when she left to visit the restroom unaccompanied, which occurred on at least one known occasion while awaiting tissue processing.

Patients may experience several side effects when using opioid analgesics, most commonly nausea and constipation. When opioids are used long-term, patients are at increased risk for developing fractures, as opioids may decrease bone mineral density by impairing the production of exogenous sex steroid hormones. ${ }^{1}$ Respiratory depression also can occur, especially when combined with alcohol and other medications such as benzodiazepines. Lastly, opioid dependence can develop in 1 week of regular use. ${ }^{1,2}$

Opioid overdose classically presents with depressed mental status, decreased tidal volume, decreased bowel sounds, miosis, and decreased respiratory rate. Pupillary size may be normal in acute opioid toxicity due to other co-ingested medications or substances. The best predictor of opioid overdose is a decreased respiratory rate, measured as fewer than 12 breaths per minute. ${ }^{1}$

If opioid overdose is suspected in the office setting, early intervention is critical. Rapid serum glucose should be obtained if a glucometer is available, as hypoglycemia can be confused with opioid toxicity and is easily correctable. If serum glucose is normal, the provider should notify emergency services. In a hospital setting, a rapid response or code can be initiated. In the office setting, dial 911. If not already in place, noninvasive continuous monitoring of the patient's pulse, oxygen saturation, and blood pressure is needed.

The provider's primary concern should be ensuring the patient is adequately ventilated and oxygenated. If the patient's respiratory rate is greater than 12 breaths per minute and oxygen saturation is greater than $90 \%$ on room air, as was the case with our patient, observe and reassess the patient frequently. If the oxygen saturation drops to less than $90 \%$ but the patient is breathing spontaneously, administer supplemental oxygen followed by naloxone. If the patient is breathing fewer than 12 breaths per minute, the airway can be maintained with the head tilt-chin lift technique while ventilating using a bag valve mask with supplemental oxygen, followed by administration of naloxone. ${ }^{1}$
Naloxone is a short-acting opioid antagonist used to treat potentially fatal respiratory depression associated with opioid overdose. It is available in intramuscular (IM), intravenous (IV), and intranasal forms. Intramuscular and IV administration are preferred due to a more rapid onset compared to intranasal. The dosage is 0.04 to $2 \mathrm{mg}$ for IM or IV formulations and $4 \mathrm{mg}$ for the intranasal formulation. ${ }^{1,3}$ The anterolateral thigh is the preferred IM injection site. Lower initial doses for the IM and IV forms generally are advisable because of the possibility of naloxone precipitating opioid withdrawal in opioid-dependent patients. Naloxone may be administered every 2 to 3 minutes until emergency personnel arrive. Repeat dosing of naloxone should be given until ventilation is greater than 12 breaths per minute while ensuring oxygen saturation is greater than $90 \%$. If there is an inadequate response after 5 to $10 \mathrm{mg}$ of naloxone administration, reconsider the diagnosis. If there is no response after naloxone administration, continue to provide respiratory support with the bag valve mask and supplemental oxygen. After the administration of naloxone, the patient should be transported to the nearest emergency department regardless of the clinical appearance, as naloxone's half-life may be shorter than the ingested opioid, requiring further observation in a monitored setting. . $^{1,3}$

We recommend that dermatologists consider keeping naloxone in their offices. The medication is easily administered and has a relatively long shelf-life of 1 to 2 years, with a $10-\mathrm{mL}$ vial of $0.4 \mathrm{mg} / \mathrm{mL}$ solution costing less than $\$ 200$ in most cases. ${ }^{3}$ Increasing cases of opioid abuse could lead to more clinical scenarios similar to what we experienced. Proper identification and management of opioid overdose is within the purview of the dermatologist and can be lifesaving.

\section{REFERENCES}

1. Stolbach A, Hoffman RS. Acute opioid intoxication in adults. UpToDate website. https://www.uptodate.com/contents/acute - opioid-intoxication-in-adults? search=acute $\% 20$ opioid $\%$ 20intoxication \%20in \%20adults\&source $=$ search_result\&selected Title $=1 \sim 150 \&$ usage_type $=$ default\&display_rank $=1$. Updated October 1, 2019. Accessed July 23, 2020.

2. Glass JS, Hardy CL, Meeks NM, et al. Acute pain management in dermatology: risk assessment and treatment. J Am Acad Dermatol. 2015;73:543-560.

3. Pruyn S, Frey J, Baker B, et al. Quality assessment of expired naloxone products from first-responders' supplies. Prehosp Emerg Care. 2018;23:647-653. 